\title{
Estimation of pulmonary artery wedge pressure from chest radiograph in patients with chronic congestive cardiomyopathy and ischaemic cardiomyopathy ${ }^{\star}$
}

\author{
HAROLD DASH, $†$ MARTIN J LIPTON, KANU CHATTERJEE, \\ WILLIAM W PARMLEY
}

From the Cardiovascular Research Institute and the Cardiovascular Division of the Department of Medicine, University of California, San Francisco, California, USA

SUMMARY In order to define the relation between chest $x$-ray findings and the level of pulmonary artery wedge pressure in patients with chronic congestive cardiomyopathy, 82 patients had chest radiographs before undergoing 92 haemodynamic studies. The studies were divided into three groups according to the level of pulmonary artery wedge pressure (PAWP) (group 1: $<15 \mathrm{mmHg}$, group 2: 15 to $24 \mathrm{mmHg}$, group 3: $\geqslant 25 \mathrm{mmHg}$.

Venous distribution, interstitial oedema, pleural effusions, left atrial enlargement, and right ventricular enlargement each occurred in less than 10 per cent of group 1 studies. Radiological abnormalities generally distinguished group 1 from group 2, but none except cardiothoracic ratio distinguished group 2 from group 3. Cardiothoracic ratio correlated best with pulmonary artery wedge pressure $(r=0.70)$. Alveolar oedema was uncommon when PAWP $\geqslant 25 \mathrm{mmHg}$, occurring in 32 per cent of group 3 studies.

Stepwise multiple linear regression analysis showed that cardiothoracic ratio, alveolar oedema, interstitial oedema, and left atrial size each contributed independently to the prediction of PAWP. The regression analysis improved the accuracy of the estimation of PAWP from the findings noted on standard chest radiographs.

A rise in left ventricular diastolic and pulmonary artery wedge pressures is a characteristic haemodynamic finding in patients with chronic congestive cardiomyopathy. However, the magnitude of the rise in pulmonary artery wedge pressure may vary widely in such patients; and, in a few, it may even be normal. Accurate determination of the level of pulmonary venous hypertension in these patients is useful for the choice of appropriate treatment, particularly for the choice of vasodilator agents which are now widely used for treatment of chronic congestive heart failure. ${ }^{1-4}$ At present, invasive haemodynamic monitoring is most commonly used to assess the severity of pulmonary venous hypertension in these patients. An accurate noninvasive method for the prediction of pulmonary artery

* This work was supported in part by a Programme Project.

† Present address: Department of Cardiology, Pennsylvania State School of Medicine, Hershey, Pennsylvania, USA.

Received for publication 28 January 1980 wedge pressure would thus be extremely useful in clinical practice.

The chest radiograph has been used to evaluate the haemodynamic status of patients with cardiac disease in the past. ${ }^{5-11}$ However, much of the data derives from patients with rheumatic mitral valvular disease, ${ }^{5-8}$ 12-14 especially mitral stenosis, and it remains to be seen that the established radiological criteria for estimating pulmonary artery wedge pressure are applicable to patients with chronic left ventricular failure. The purpose of this study, therefore, was to assess the value of chest radiographic findings for the estimation of pulmonary artery wedge pressure in patients with chronic left ventricular failure.

\section{Subjects and methods}

All patients undergoing haemodynamic monitoring for symptoms of chronic congestive heart failure at 
the University of California, San Francisco, between January 1975 and January 1979, were evaluated for eligibility in the study. Patients were accepted into the study if they met the following criteria: (1) symptoms of heart failure of more than one month's duration; (2) a standard posteroanterior and lateral chest radiograph performed within 72 hours before haemodynamic evaluation; (3) a stable clinical status without change in symptomatology or therapeutic regimen during the interval between radiological and haemodynamic assessment. Patients with valvular or congenital heart disease or hypertrophic cardiomyopathy were excluded from the study. Of a total of 128 patients, 56 met the above criteria. Thirty-eight patients undergoing 46 haemodynamic studies were evaluated retrospectively. Eighteen patients undergoing 20 studies were then evaluated prospectively. An additional 26 patients without symptoms of heart failure who underwent right heart catheterisation during the course of evaluation for chest pain syndromes served as a control group. Twenty were evaluated retrospectively and six prospectively. Thus, the retrospective group consisted of 24 patients undergoing 26 haemodynamic studies.

All patients had chronic exertional dyspnoea of over one month's duration. Those who had biplane left ventricular angiography had an ejection fraction $\leqslant 0.40$. All had, in addition, at least two of the following characteristics: (1) a third heart sound, (2) raised jugular venous pressure, (3) a sustained laterally displaced left ventricular impulse, (4) chronic digitalis or diuretic requirements, (5) pulmonary artery wedge pressure $\geqslant 15 \mathrm{mmHg}$, (6) cardiac index $\leqslant 2 \cdot 21 / \mathrm{min}$ per $\mathrm{m}^{2}$. Fifty-three of the patients with congestive cardiomyopathy were in New York Heart Association class III or IV heart failure at the time of the study; three were in class II. Two of the patients in class IV had improved to class II at the time of their second study.

\section{PATIENT POPULATION}

The mean age of patients with cardiomyopathy was $59 \pm 13( \pm 1 \mathrm{SD})$ years compared with $55 \pm 12$ years for the patients in the control group. The difference was not statistically significant. The mean ages of the patients and controls in the retrospective and prospective groups were similar to the groups as a whole.

Both cardiomyopathy and control populations were predominantly male, with $39(70 \%)$ men in the former and $21(81 \%)$ men in the latter (NS). The sex ratios of the cardiomyopathy and control patients in the retrospective and prospective groups were also similar to those of the overall groups and not statistically different from one anotner.

Thirty-two $(57 \%)$ of the heart failure patients had cardiomyopathy secondary to coronary artery disease, as determined by coronary angiography or a documented history of multiple myocardial infarctions (ischaemic cardiomyopathy). Three had alcoholic cardiomyopathy, one was diabetic with normal coronary arteries, and one was a female carrier for the X-linked disorder, Duchenne's muscular dystrophy. Thirteen $(23 \%)$ had a congestive cardiomyopathy of undetermined aetiology. Because of similar clinical and haemodynamic presentations in patients with ischaemic cardiomyopathy and in patients with congestive cardiomyopathy, results were analysed together.

Of the control population, 21 had coronary artery disease and five had atypical chest pain with normal coronary arteries.

\section{HAEMODYNAMIC EVALUATION}

Right heart catheterisation with a Swan-Ganz catheter was performed in the cardiac catheterisation laboratory or the coronary care unit after the patients gave informed consent. Pulmonary artery and pulmonary artery wedge pressures were measured with the patient lying supine using a Statham P231d or P231a strain gauge. Zero reference level was at the mid-axillary line.

The patients evaluated retrospectively were divided into three groups according to their mean pulmonary artery wedge pressure: group 1 included those with pulmonary artery wedge pressure $\leqslant 15 \mathrm{mmHg}$; group 2 included those with pulmonary artery wedge pressure 15 to $24 \mathrm{mmHg}$; and group 3 included those with pulmonary artery wedge pressure $\geqslant 25 \mathrm{mmHg}$.

\section{RADIOLOGICAL INDICES}

All chest radiographs were obtained in the Radiology Department. Posteroanterior and lateral projections were obtained at end inspiration with an $x$-ray source-to-film distance of six feet at approximately $140 \mathrm{kV}$.

Cardiac size was evaluated using the cardiothoracic ratio, which was measured as the maximum transverse diameter of the heart divided by the maximum thoracic width. ${ }^{15}$ In addition, the presence of left and right ventricular and left atrial chamber enlargement was recorded. Definitions of chamber enlargement were based on standard criteria. ${ }^{15}$

Pulmonary venous flow redistribution was defined as the presence of a disparity between the calibre of the upper and lower lobe pulmonary vessels with distention of the upper vessels. ${ }^{1012}$ Interstitial oedema was recognised as perivascular 
or peribronchial cuffing, or Kerley's septal lines. ${ }^{16}$ Alveolar oedema was diagnosed as a hazy mottling of small more discrete densities, some confluent, without the linear appearance characteristics of interstitial oedema, and with poorly defined borders. ${ }^{77}$ Pleural effusions were noted. Pulmonary venous flow redistribution, interstitial oedema, alveolar oedema, and pleural effusion were termed as "fluid abnormalities".

Finally, the presence or absence of hilar and pulmonary artery enlargement was recorded. ${ }^{6}$

Chest radiographs were interpreted by one observer without knowledge of the clinical or haemodynamic status of the patients. To assess intraobserver variability, 20 chest radiographs were reinterpreted blindly three months after initial radiological assessment.

\section{STATISTICAL ANALYSIS}

The data were processed using the statistical packages provided by the BMDP Biomedical Computer Programs P-series. ${ }^{18}$ Comparisons of groups of data consisting of continuous variables (as in Table 1 and Fig. 1) were done with t tests or analysis of variance followed by a Student-NewmanKeuls multiple comparison test. Groups of discrete variable (see Fig. 2 and 3) were evaluated with nonparametric statistics, including the Mann-Whitney and Kruskal-Wallis tests.

Table Haemodynamic results

\begin{tabular}{|c|c|c|c|}
\hline \multirow{2}{*}{$\begin{array}{l}\text { Group } \\
1(\mathrm{~N}=25) \\
2(2(\mathrm{~N}=13) \\
3(\mathrm{~N}=28)\end{array}$} & \multicolumn{2}{|c|}{$P A W P(m m H g)$} & $P A P(m m H g)$ \\
\hline & $\begin{array}{l}9 \cdot 4 \pm 2 \cdot 6^{\star} \\
19 \cdot 7 \pm 3 \cdot 2 \\
30 \cdot 8 \pm 4 \cdot 5\end{array}$ & $\left\{\begin{array}{l}\mathrm{p}<0.001 \\
\mathrm{p}<0.001\end{array}\right.$ & $\left.\begin{array}{l}15.6 \pm 2.6 \\
30.0 \pm 6.0 \\
40.4 \pm 6.0\end{array}\right\} \mathrm{p}<0.001$ \\
\hline
\end{tabular}

* Mean \pm 1 SD.

Abbreviations: PAWP = pulmonary artery wedge pressure;

PAP = pulmonary artery pressure; $\mathrm{N}=$ number of studies;

$1=$ studies with PAWP $<15 ; 2=$ studies with PAWP 15 to 24 ;

$3=$ studies with PAWP $\geqslant 25$.

Bivariate linear regression and stepwise multiple linear regression analysis were also used to relate the radiological data to the haemodynamics (Fig. 4). The stepwise regression program evaluated all the variables presented to it and selected only those radiological variables which contributed independently to a significant degree to the prediction of pulmonary artery wedge pressure. Multiple correlation coefficients ( $r$ ), fraction of variance explained $\left(\mathbf{R}^{2}\right)$, levels of significance for each variable and the overall regression, and standardised regression coefficients were obtained at each step. The latter are normalised values which take into account the different units and variances of the variables.

\section{Results}

INTRAOBSERVER VARIABILITY

Analysis of intraobserver variability showed that interpretation of the chest radiographs was highly consistent, except for the assessment of hilar and pulmonary artery size. The initial mean cardiothoracic ratio of the 20 radiographs read twice was $0.58 \pm 0.11$ ( $\pm 1 \mathrm{SD}$ ) and the subsequent value $0 \cdot 58 \pm 0 \cdot 10$. All paired cardiothoracic ratios were consistent to 2 per cent except two pairs which were accurate to 6 and 5 per cent of one another. Paired

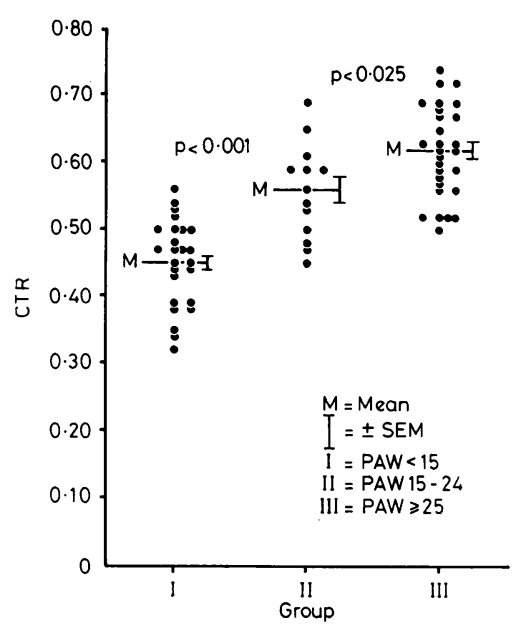

Fig. 1 Plotted are the means and individual values for the cardiothoracic ratios in each group of patients. The mean value of group 2 was significantly higher $(p<0.001)$ than group 1, and that of group 3 significantly higher than group $2(p<0.025)$.

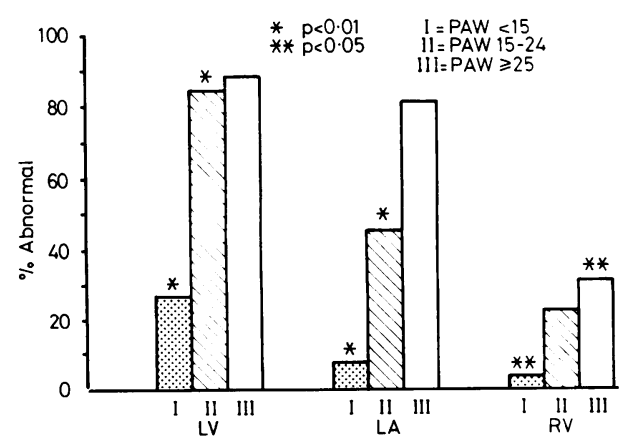

Fig. 2 Presented are histograms showing the percentage of findings with left ventricular $(L V)$ enlargement, left atrial (LA) enlargement, and right ventricular ( $R V)$ enlargement in each group. 
estimates of the pulmonary venous pattern, presence of interstitial and alveolar oedema, pleural effusions, left and right ventricular size, and left atrial size were all consistent in at least 90 per cent of cases. On the other hand, 20 per cent of estimates of pulmonary artery size and 40 per cent of estimates of hilar size were inconsistent. Given the high variability

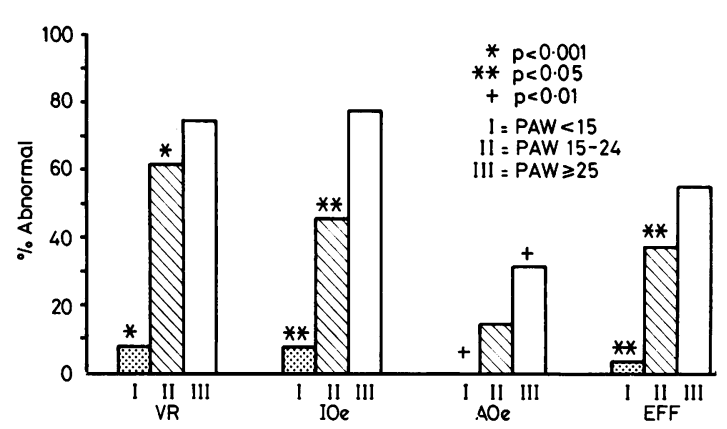

Fig. 3 Presented are histograms showing the percentage of findings with venous redistribution (VR), interstitial oedema $(I O e)$, alveolar oedema $(A O e)$, and pleural effusions (EFF) in each group.

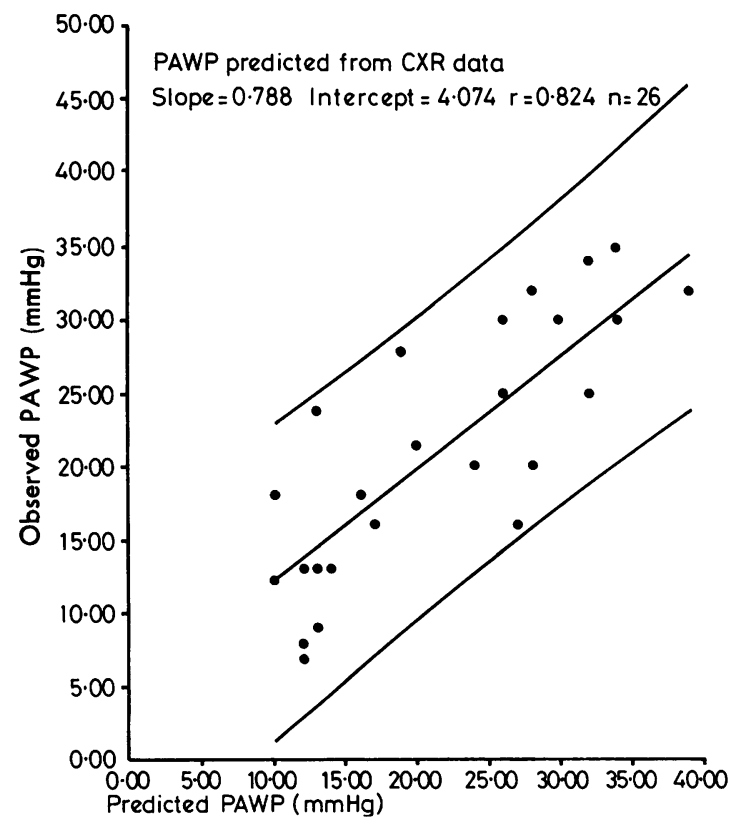

Fig. 4 Plotted is the predicted versus the observed pulmonary artery wedge pressures in the prospective group of studies, the resultant linear regression formula ( $\operatorname{slope}=0.79, r=0.82$ ), and the 95 per cent confidence limits. in the assessment of pulmonary artery and hilar size, these two radiological variables were excluded from the analysis relating the chest radiographic findings to pulmonary artery wedge pressure. All the other chest $x$-ray indices described above form the bases for the subsequent results.

\section{Retrospective results}

\section{HAEMODYNAMIC FINDINGS}

Of the 66 studies done retrospectively, the mean pulmonary artery wedge pressure was $26 \cdot 0 \pm 8 \cdot 2$ ( $\pm 1 \mathrm{SD}) \mathrm{mmHg}$ for the cardiomyopathy group and $9.1 \pm 2.5 \mathrm{mmHg}$ for the control population $(p<0.001)$. The Table lists the resultant mean pulmonary artery wedge and pulmonary artery pressures after the patients and controls were divided into three groups according to their pulmonary artery wedge pressure. It is, therefore, not surprising that the haemodynamics of each group were highly statistically different $(p<0.001)$ group 1 vs. 2 , and 2 vs. 3 for pulmonary artery wedge and pulmonary artery pressures.

\section{RADIOLOGICAL ASSESSMENT OF CARDIAC ENLARGEMENT AND CHAMBER SIZE}

Heart size alone, as estimated by cardiothoracic ratio, correlated well with pulmonary artery wedge pressure $(r=0 \cdot 70)$. However, the 95 per cent confidence limits were wide, making the prediction of an individual patient's pulmonary artery wedge pressure somewhat uncertain. This difficulty is illustrated in Fig. 1, in which the cardiothoracic ratios for all the retrospective studies are plotted according to group. Mean cardiothoracic ratio for the group 1 patients was $0.45 \pm 0.06$; for the group 2 patients, $0.57 \pm 0.08$; and for the group 3 patients, $0.62 \pm 0.08$ ( $p<0.0011$ vs. $2, p<0.0252$ vs. 3 ). It can be seen, however, that the overlap among the groups is great enough to make the classification of individual patients difficult. Generally, a cardiothoracic ratio $\geqslant 0.60$ was predictive of pulmonary artery wedge pressure $\geqslant 25 \mathrm{mmHg} ; 77$ per cent of studies with a cardiothoracic ratio $\geqslant 0.60$ occurred when pulmonary artery wedge pressure was $\geqslant 25 \mathrm{mmHg}$. On the other hand, the sensitivity of such an abnormal cardiothoracic ratio for a pulmonary artery wedge pressure $\geqslant 25 \mathrm{mmHg}$ was low, occurring in 58 per cent of the group 3 patients. Normal cardiothoracic ratio $(\leqslant 0.50)$ suggested normal pulmonary venous pressure; 88 per cent of studies with cardiothoracic ratio $\leqslant 0.50$ had pulmonary artery wedge pressure $<15 \mathrm{mmHg}$. Only 68 per cent of all studies, however, with pulmonary artery wedge pressure $<15 \mathrm{mmHg}$ had a normal cardiothoracic ratio. 
Fig. 2 depicts the presence of chamber enlargement in the three groups. Left ventricular enlargement occurred in 27 per cent of group 1 patients, whereas left atrial or right ventricular enlargement occurred in less than 10 per cent of group 1 patients. Though left ventricular and left atrial enlargement were statistically more frequent when pulmonary artery wedge pressure was raised $(p<0.01)$, no chamber assessment discriminated those with mild to moderate (group 2) from those with severe (group 3) rises in pulmonary artery wedge pressure.

Fig. 3 shows the presence of "fluid abnormalities" in the three groups. Fluid abnormalities were uncommon when pulmonary artery wedge pressure was $15 \mathrm{mmHg}$. Venous redistribution, interstitial oedema, and pleural effusions occurred in less than 10 per cent of group 1 patients, while alveolar oedema occurred in none. Venous abnormalities $(\mathrm{p}<0.001)$, interstitial oedema $(\mathrm{p}<0.05)$, and pleural effusions $(p<0.05)$ all distinguished group 1 from group 2. None of the radiological types of fluid abnormalities, however, were statistically more frequent when the group 3 studies were compared with those in group 2. Alveolar oedema was present rarely even when pulmonary capillary wedge pressure was $>25 \mathrm{mmHg}$, when it was present in 32 per cent of studies.

Multiple linear regression analysis showed that radiological assessment of cardiothoracic ratio, alveolar oedema, interstitial oedema, and left atrial size (in that order of importance) all independently contributed to the estimation of pulmonary artery wedge pressure $r=0.82$ ). Cardiothoracic ratio accounted for 48 per cent of the variance explained $\left(R^{2}\right)$; alveolar oedema 11 per cent, and interstitial oedema and left atrial size, each 4 per cent. The standardised regression coefficients of each variable were similar, however, indicating that all four variables were important for the estimation of pulmonary artery wedge pressure from the chest radiograph. The regression formula was:

$$
\begin{gathered}
\text { PAWP }=-2 \cdot 6 \pm 21 \cdot 1(\mathrm{CTR})+5 \cdot 5(\mathrm{AOe}) \\
+5 \cdot 4(\mathrm{IOe})+6 \cdot 3(\mathrm{LA})
\end{gathered}
$$

where PAWP = pulmonary artery wedge pressure and is measured in $\mathrm{mmHg}, \mathrm{CTR}=$ cardiothoracic ratio, $\mathrm{AOe}=$ alveolar oedema, $\mathrm{IOe}=$ interstitial oedema, and LA = left atrial enlargement. AOe, IOe, or LA were given a value of " 1 " if the particular abnormality was present and " 0 " if absent.

\section{Prospective results}

In order to test the validity of the derived stepwise linear regression formula, the formula was then applied to the radiological data of the studies obtained prospectively. The formula was used to derive predicted pulmonary artery wedge pressures from the prospective radiological data. The predicted pressures were then correlated with actual pressures. The results are plotted in Fig. 4. The correlation is excellent, with $r=0.82$, a value similar to that obtained from the retrospective data.

\section{Discussion}

Previous investigations documented the value of the chest radiographic findings of pulmonary venous flow redistribution, interstitial oedema, alveolar oedema, and pulmonary arterial enlargement in the noninvasive estimation of the haemodynamics in patients with rheumatic mitral valvular dis-

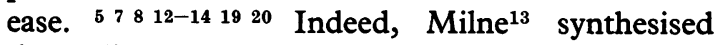
the available data and presented specific radiological criteria for the estimation of both mean pulmonary artery and pulmonary artery wedge pressures in patients with mitral stenosis.

In the present study an attempt was made to evaluate the predictive value of various radiological findings for the estimation of pulmonary venous pressure in patients with chronic congestive heart failure who did not have valvular heart disease. Our findings document that the presence of most chest radiographic abnormalities in patients with chronic congestive cardiomyopathy and ischaemic cardiomyopathy indicates raised pulmonary artery wedge pressure (Fig. 1 to 3). Alveolar oedema was conspicuously absent in patients with normal pulmonary artery wedge pressure. Left atrial and right ventricular enlargement also occurred rarely $(<10 \%)$ when pulmonary venous pressure was normal. The only radiological abnormality common despite a normal pulmonary artery wedge pressure was left ventricular enlargement $(27 \%$ of group 1 studies). This finding may reflect the nature of our control patients: many had prior myocardial infarction and/or hypertension.

Our observations in patients with chronic congestive cardiomyopathy are at variance with those in patients with acute heart failure secondary to myocardial infarction as reported by Kostuk et al. ${ }^{21}$ who showed that in the setting of acute myocardial infarction, the chest radiograph predicted accurately the pulmonary artery wedge pressure in only 43 per cent of patients, underestimated it in 24 per cent, and overestimated it in 33 per cent. We found that radiographic analysis in chronic heart failure rarely overestimated pulmonary artery wedge pressure. Rapidly changing haemodynamics in patients with acute myocardial infarction may account for this discrepant result. 
Although the presence of "fluid abnormalities" in patients with chronic congestive heart failure in our study was useful to separate patients with normal pulmonary artery wedge pressure from those with raised pulmonary artery wedge pressure, the findings did not help in distinguishing patients with moderate increases in pulmonary artery wedge pressure (group 2) from those with severe increases (group 3) (Fig. 2). Our findings differ from those of Turner et al. ${ }^{11}$ who estimated pulmonary haemodynamics from the chest radiographic analysis of fluid abnormalities and pulmonary arterial size. However, in their study haemodynamic and radiological correlations are not presented other than several individual cases, and the patient population is not identified. Moreover, in our study we have shown, as have others previously, ${ }^{9}$ that the radiological estimation of pulmonary size may be in error.

Of the fluid abnormalities, alveolar oedema was the most specific predictor of a raised pulmonary artery wedge pressure in our study. No group 1 patient had alveolar oedema. Yet alveolar oedema was also uncommon, despite severe increases in pulmonary artery wedge pressure. ${ }^{18}$ Some authors $^{11} 15$ have suggested that transudation of oedema into the alveoli does not occur until the pulmonary artery wedge pressure reaches 30 to $35 \mathrm{mmHg}$. Of our studies in which pulmonary artery wedge pressure was at least $30 \mathrm{mmHg}$, alveolar oedema was present in 30 per cent. One possible explanation for infrequent pulmonary oedema despite severe rises in pulmonary venous pressure is that, during chronic heart failure, lymphatic hypertrophy and hyperplasia and changes in the pulmonary interstitium allow for a more efficient removal of oedema from the lungs than in acute heart failure. ${ }^{22}$

Cardiomegaly as assessed by cardiothoracic ratio proved to be the single most important predictor of pulmonary artery wedge pressure in our study (Fig. 1). This finding, however, is contrary to the data reported in the setting of acute myocardial infarction by $\mathrm{McHugh}$ et al. ${ }^{23}$ and Kostuk et al. ${ }^{21}$ The different findings may relate to cardiovascular adaptation to heart failure. Gradual left ventricular dilatation is the usual pathophysiological response to an increase in left ventricular filling pressure and a reduction in myocardial contractility. ${ }^{24}$ Consequently, cardiomegaly is more likely to be present in chronic rather than acute left ventricular failure. In fact, Kostuk et al. ${ }^{21}$ noted that those patients with radiographic cardiomegaly nearly always had raised pulmonary capillary wedge pressure.

Our data are in agreement with those of Harlan et $a i^{25}$ who found that radiological estimate of heart volume was the most important clinical descriptor of a group of patients with ischaemic cardiomyopathy. Though Yatteau et al. ${ }^{26}$ found normal heart size in 27 per cent of their patients with ischaemic cardiomyopathy, 60 per cent of their patients were in New York Heart Association class I or II heart failure, whereas only 6 per cent of our patients were in class I or II.

Although in the present study cardiothoracic ratio correlated best with pulmonary artery wedge pressure $(r=0 \cdot 70)$, the accuracy of the ratio in predicting the level of increase in pulmonary artery wedge pressure in individual patients was low. In many patients the cardiothoracic ratio could not be used to estimate the pulmonary artery wedge pressure with enough precision to be clinically useful.

The present study indicates that a combination of radiological variables might more accurately predict the level of pulmonary artery wedge pressure. The stepwise multiple linear regression formula, using the variables cardiothoracic ratio, alveolar oedema, interstitial oedema, and left atrial size, predicted pulmonary capillary wedge pressure fairly accurately in patients with ischaemic as well as congestive cardiomyopathy $(r=0.82)$. The regression equation derived from the retrospective data was used prospectively to estimate pulmonary artery wedge pressure in 26 studies, and a close estimation of pulmonary artery wedge pressure was possible in most patients $(r=0.82)$. It needs to be emphasised, however, that the slope of the regression line in the prospective evaluation was 0.79 , indicating that the radiological findings tended to underestimate the pulmonary artery wedge pressure to some extent (Fig. 4). This is not surprising given that alveolar oedema occurred infrequently, yet was the second most important predictor for raised pulmonary artery wedge pressure in our study. Fig. 5 illustrates the difficulty that exists in the radiological estimation of the pulmonary haemodynamics in patients with chronic congestive cardiomyopathy. The two patients whose $x$-rays are shown in Fig. 5 both had identical haemodynamic findings with cardiac indices of $1.51 / \mathrm{min}$ per $\mathrm{m}^{2}$ and pulmonary artery wedge pressure of $32 \mathrm{mmHg}$. Both patients had gross cardiomegaly and left ventricular and atrial enlargement, yet only the patient in Fig. 5A had pulmonary venous flow redistribution, interstitial oedema, and alveolar oedema. Using traditional criteria, a radiologist might estimate that the patient in Fig. 5B had normal or minimally raised left ventricular filling pressures; using the stepwise linear regression formula, the estimated pulmonary artery wedge pressure is $23 \mathrm{mmHg}$, and slightly 

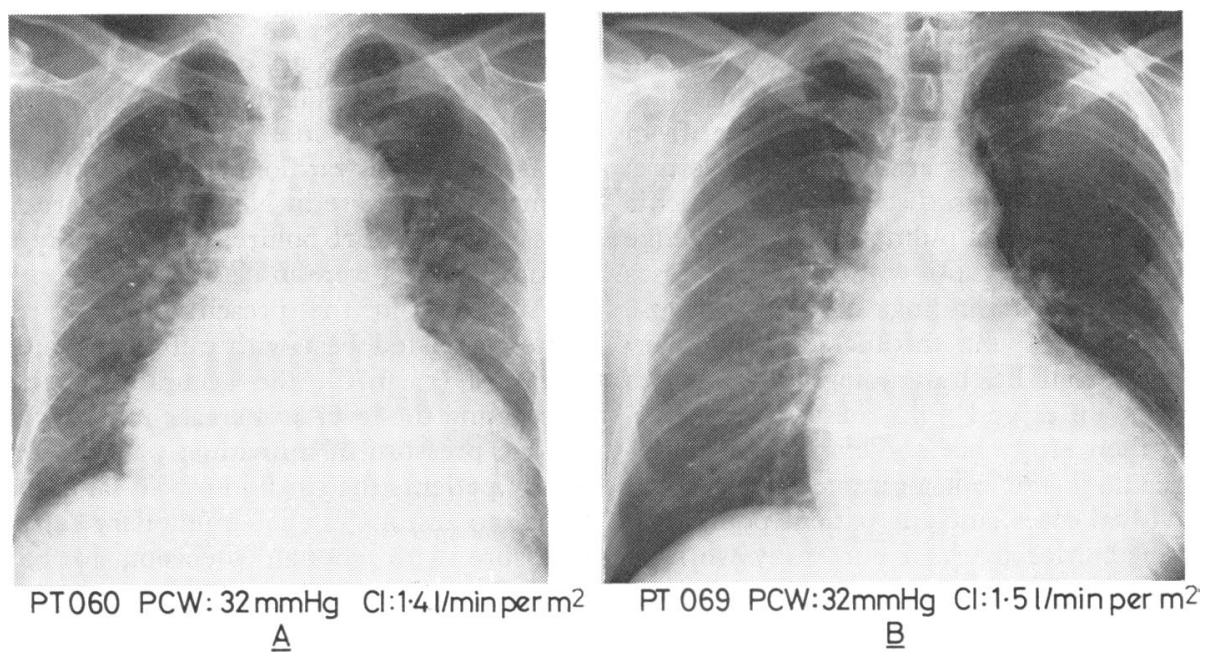

Fig. 5 Shown are chest radiographs from two patients with identical haemodynamics (pulmonary artery wedge pressure $=32 \mathrm{mmHg}$ and cardiac index $=1.5 \mathrm{l} / \mathrm{min}$ per $\mathrm{m}^{2}$ ) and different radiographic findings.

underestimates the true pulmonary artery wedge pressure, though it is still grossly abnormal.

In conclusion, our study has shown that, though chest radiographic abnormalities are helpful to identify the presence of raised pulmonary venous pressure in patients with ischaemic and congestive cardiomyopathy, the prediction of the level of pulmonary venous pressure from any single radiological variable is not possible. Multiple stepwise linear regression analysis improves the predictive value of the radiological variables for the estimation of pulmonary artery wedge pressure. A prospective study involving a much larger group of patients will be needed to evaluate further the value of the regression analysis for the estimation of pulmonary artery wedge pressure in these patients.

The authors thank Drs James Ostlund and Stanton Glantz for their assistance with the statistical analysis.

\section{References}

1 Kovick RB, Tillisch JH, Berens SC, Bramowitz AD, Shine KI. Vasodilator therapy for chronic left ventricular failure. Circulation 1976; 53: 322-8.

2 Franciosa JA, Mikulic E, Cohn JN, Jose E, Fabi A. Hemodynamic effects of orally administered isosorbide dinitrate in patients with congestive heart failure. Circulation 1974; 50: 1020-4.

3 Chatterjee K, Parmley WW, Massie B, et al. Oral hydralazine therapy for chronic refractory heart failure. Circulation 1976; 54: 879-83.
4 Chatterjee K, Drew D, Parmley WW, Klausner SC, Polansky J, Zacherle B. Combination vasodilator therapy for severe chronic congestive heart failure. Ann Intern Med 1976; 85: 467-70.

5 Grainger RC. Interstitial pulmonary oedema and its radiological diagnosis: a sign of pulmonary venous and capillary hypertension. $\mathrm{Br} \mathcal{f}$ Radiol 1958; 31: 201-17.

6 Lavender JP, Doppman J. The hilum in pulmonary venous hypertension. $\mathrm{Br} \mathcal{F}$ Radiol 1962; 35: 303-13.

7 Steiner RE. Radiology of pulmonary circulation. Am $\mathcal{F}$ Roentgenol 1964; 91: 249-64.

8 Simon M, Sasahara AA, Cannilla JE. The radiology of pulmonary hypertension. Semin Roentgenol 1967; 2: 368-88.

9 Short DS. Radiology of the lung in left heart failure. Br Heart $\mathcal{F} 1956$; 18: 233-40.

10 Simon $M$. The pulmonary vessels in incipient left ventricular decompensation. Circulation 1961; 24: 185-90.

11 Turner AF, Lau FYK, Jacobson G. A method for the estimation of pulmonary venous and arterial pressures from the routine chest roentgenogram. Am $\mathcal{F}$ Roentgenol 1972; 116: 97-106.

12 Simon $M$. The pulmonary veins in mitral stenosis. F Fac Radiologists 1956; 9: 25-32.

13 Milne ENC. Physiological interpretation of the plain radiograph in mitral stenosis, including a review of criteria for the radiological estimation of pulmonary arterial and venous pressures. $\mathrm{Br} \mathcal{F}$ Radiol 1963; 36: 902-13.

14 Chen JTT, Behar VS, Morris JJ Jr, McIntosh HD, Lester RG. Correlation of roentgen findings with hemodynamic data in pure mitral stenosis. Am $\mathfrak{f}$ Roentgenol 1968; 102: 280-92. 
15 Jefferson K Rees S. Clinical cardiac radiology. London: Butterworth, 1973: 3-25, 84-94.

16 Chait A. Interstitial pulmonary edema. Circulation 1972; 45: 1323-30.

17 Meszaros WT. Lung changes in left heart failure. Circulation 1973; 47: 859-71.

18 Dixon WJ, Brown MB, eds. BMDP-77, Biomedical computer programs, P-series. Rev. ed. Berkeley: University of California Press, 1978: 1-154, 167-98, 375-417.

19 Lavender JP, Doppman J, Shawdon H, Steiner RE. Pulmonary veins in left ventricular failure and mitral stenosis. Br F Radiol 1962; 35: 293-302.

20 Salzmann C, Sutton GC, Chatterjee K, Kerr IH, Miller GAH. Assessment of homograft replacement of mitral valve by chest radiographs. Br Heart $\mathcal{f}$ $1972 ; 34: 121-8$.

21 Kostuk W, Barr JW, Simon AL, Ross J Jr. Correlations between the chest film and hemodynamics in acute myocardial infarction. Circulation 1973; 48: 624-32.

22 Harris $\mathrm{P}$, Heath $\mathrm{D}$. The human pulmonary circulation 2nd ed. Edinburgh: Churchill Livingstone, 1977: 341-3, 361-2.
23 McHugh TJ, Forrester JS, Adler L, Zion D, Swan HJC. Pulmonary vascular congestion in acute myocardial infarction: hemodynamic and radiologic correlations. Ann Intern Med 1972; 76: 29-33.

24 Mason DT, Spann JF Jr, Zelis R, Amsterdam EA. Alterations of hemodynamics and myocardial mechanics in patients with congestive heart failure: pathophysiologic mechanisms and assessment of cardiac function and ventricular contractility. Prog Cardiovasc Dis 1970; 12: 507-57.

25 Harlan WR, Oberman A, Grimm R, Rosati RA. Chronic congestive heart failure in coronary artery disease: clinical criteria. Ann Intern Med 1977; 86: 133-8.

26 Yatteau RF, Peter RH, Behar VS, Bartel AG, Rosati RA, Kong Y. Ischemic cardiomyopathy: the myopathy of coronary artery disease. Am $f$ Cardiol $1974 ; 34$ : 520-5.

Requests for reprints to Professor Kanu Chatterjee, Room 1186 Moffitt, University of California, San Francisco, California 94143, USA. 